\title{
FOTOBIOMODULAÇÃO NO TRATAMENTO DE NEURALGIA PÓS-HERPÉTICA: CASO CLÍNICO
}

\author{
PHOTOBIOMODULATION IN THE TREATMENT OF POST-HERPETIC \\ NEURALGIA: A CLINICAL CASE
}

\author{
Erick Giovanni Reis Silva ${ }^{1}$ \\ Emilia Angela Lo Schiavo Arisawa ${ }^{2}$ \\ Rodrigo Alvaro Brandão Lopes-Martins ${ }^{3}$ \\ Raimundo Nonato Silva Gomes ${ }^{4}$ \\ Renata Amadei Nicolau ${ }^{5}$ \\ Ana Paula Pinto ${ }^{6}$
}

Resumo: $O$ presente caso clínico teve por objetivo avaliar a ação da fotobiomodulação (PBM) no tratamento de paciente com diagnóstico de Neuropatia Pós-Herpética (NPH), visando o alívio da dor e a melhora da qualidade de vida. Para a PBM utilizou-se um cluster com três lasers de GaAlAs, com os parâmetros: $660 \mathrm{~nm}$, $100 \mathrm{~mW}$, irradiação pontual, $40 \mathrm{~s} /$ ponto, $4 \mathrm{~J} / \mathrm{cm} 2$ por ponto e total de 10 sessões. Para aferição da dor foram utilizadas a Escala Visual Analógica da Dor (EVA) e Algometria, e a qualidade de vida, com aplicação do Questionário de Avaliação da Qualidade de Vida -SF-36. Os dados obtidos foram submetidos à análise descritiva e ao teste estatístico T pareado, com nível de significância de $5 \%(p<0,05)$. Os resultados deste estudo demonstraram redução da dor quando comparadas a $1^{\mathrm{a}}$ à $10^{\mathrm{a}}$ sessão, tanto pela EVA quanto pela Algometria. Com relação à análise da qualidade de vida, foi observada uma melhora significativa em todos os oito domínios avaliados. Portanto, conclui-se que a PBM foi eficaz no tratamento da NPH visto que houve redução da dor e melhora na qualidade de vida. Esses resultados são importantes e podem nortear novas pesquisas clínicas que objetivem o emprego da PBM no tratamento da $\mathrm{NPH}$.

Palavras-chave: Neuralgia pós herpética. Qualidade de vida. Fotobiomodulação. Laser. Escala visual analógica.

Abstract: The present clinical case aimed to evaluate the action of photobiomodulation (PBM) in the treatment of a patient diagnosed with Post-Herpetic Neuropathy (PHN), aiming at pain relief and improvement of the quality of life. For the PBM, a cluster with three GaAlAs lasers was used, with the parameters: $660 \mathrm{~nm}, 100$ $\mathrm{mW}$, point irradiation, 40 seconds per point, $4 \mathrm{~J} / \mathrm{cm} 2$ per point and a total of 10 sessions. To measure the pain, the Visual Analog Pain Scale (VAS) and Algometry were used, as well as the quality of life, applying the Quality of Life Assessment Questionnaire - SF-36. The data obtained was submitted to descriptive analysis and to the paired T statistical test, with a significance level of $5 \%(p<0.05)$. The results of this study showed pain reduction compared to the 1 st to the 10th sessions, both by

\footnotetext{
${ }^{1}$ Mestre em Engenharia Biomédica - Univap, E-mail: erickgiovannirs@yahoo.com.br.

2 Docente da Universidade do Vale do Paraíba - Univap, E-mail: mirelarisawa@hotmail.com.

3 Universidade Brasil, E-mail: ralopesmartins@gmail.com.

${ }^{4}$ Docente da Universidade Federal do Rio de Janeiro -UFRJ, E-mail: raigomezz19@gmail.com.

${ }^{5}$ Doutora em Ciências Experimentais Aplicada à Biomedicina, E-mail: renatanicolau@hotmail.com.

6 Doutoranda em Engenharia Biomédica pela Universidade do Vale do Paraíba - Univap, E-mail: apaula@outlook.com.br.
} 
VAS and Algometry. Regarding the analysis of the quality of life, a significant improvement was observed in all eight domains evaluated. Therefore, it is concluded that PBM was effective in the treatment of PHN, since there was a reduction in pain and an improvement in the quality of life. These results are important and may guide new clinical research aimed at the use of PBM in the treatment of PHN.

Key words: Post-herpetic neuralgia. Quality of life. Photobiomodulation. Laser. Visual analog pain scale.

Data de submissão: 26.03 .2020

Data de aprovação: 10.05.2021

\section{Identificação e disponibilidade:}

(https://revista.univap.br/index.php/revistaunivap/article/view/2494,

http://dx.doi.org/10.18066/revistaunivap.v27i56.2494.

\section{INTRODUÇÃO}

A neuralgia, sintoma mais comum em pacientes acometidos por Herpes zoster $(\mathrm{HZ})$, é caracterizada por dor neuropática crônica no trajeto do nervo afetado, com persistência mínima de um mês e início entre um e seis meses após a cura das erupções cutâneas, podendo durar anos. A incidência de neuralgia pós-herpética (NPH) varia entre $10 \%$ e $20 \%$ em adultos imunocompetentes (PORTELLA; SOUZA; GOMES, 2013).

O vírus Varicella-zoster (VVZ) é um Herpesvírus que causa a varicela e persiste de forma latente no sistema nervoso após um quadro de infecção primária. A reativação do $\mathrm{V} V \mathrm{em}$ um nervo craniano ou no gânglio dorsal da raiz, com propagação ao longo do nervo sensorial para o dermátomo, leva a manifestações cutâneas dolorosas, condição essa denominada Neuralgia pós-herpética (NPH) (COELHO et al., 2014).

$\mathrm{O}$ quadro clínico da $\mathrm{HZ}$ é, quase sempre, típico. A maioria dos indivíduos refere dores neurálgicas, além de parestesias, ardor e prurido locais antecedendo as lesões cutâneas, acompanhado de febre, cefaleia e mal-estar. A lesão elementar apresentase como uma vesícula sobre base eritematosa. A erupção é unilateral, raramente ultrapassando a linha mediana do corpo, seguindo o trajeto de um nervo e surge de modo gradual, levando de 2 a 4 dias para se estabelecer (BRASIL, 2019).

Devido à elevada frequência da NPH, essa complicação se destaca por afetar diretamente a qualidade de vida do indivíduo acometido. Sua incidência é de grande variação, dependendo muito da idade e fatores que corroboram para diminuição do sistema imunológico, sendo que $5 \%$ dos pacientes têm menos de 60 anos, $10 \%$ encontram-se entre 60 e 69 anos e $20 \%$ acometem indivíduos com mais de 80 anos (YAWN et al., 2013).

Atualmente, o tratamento convencional é realizado com a administração de medicamento antiviral, sendo os mais comuns o aciclovir, valaciclovir e o fanciclovir. Estes fármacos têm sua eficácia comprovada na redução na formação de novas lesões, sem muitos efeitos sobre a dor aguda (CHEN et al., 2014, BRASIL, 2019; LE; ROTHBERG, 2019). Considerando a dificuldade encontrada para tratamento da NPH, torna-se desafiadora e necessária uma intervenção multiprofissional, incluído diferentes fármacos, procedimentos e terapias adjuvantes não farmacológicas (OLIVEIRA et al., 2016). 
Os indivíduos que sofrem com a NPH apresentam qualidade de vida reduzida, visto que em vários casos, a dor é incapacitante, limitando suas atividades laborais e do cotidiano, interferindo em diversos âmbitos da vida dos pacientes, inclusive no aspecto psicológico, com desenvolvimento de depressão (YOUNGER; MCCUE; MACKEY, 2009; DING et al., 2017).

A fotobiomodulação (PBM) se apresenta como nova ferramenta terapêutica para tratamento da NPH, tendo sido empregada com sucesso na prática clínica e ciências da saúde, pelas ações anti-inflamatória, analgésica, antiedematosa e por favorecer o processo de reparo tecidual. PBM tem demonstrado ser uma opção de tratamento efetiva, não invasiva, não medicamentosa e segura, pelos resultados relatados em outras afecções que comprovaram sua ação na redução da dor, indicando seu potencial para tratamento de pacientes acometidos pela NPH (CAVALCANTI et al., 2016; SANTOS et al., 2018; GOMES, 2018; NEVES et al., 2020).

Considerando o exposto, o objetivo deste caso clínico foi avaliar o impacto da fotobiomodulação na dor e na qualidade de vida de um indivíduo portador de Neuralgia pós-herpética.

\section{METODOLOGIA}

\section{Aspectos éticos e legais}

O projeto de pesquisa foi submetido à Plataforma Brasil e, após sua aprovação (CEP- 3.267.653, CAAE: 08945719.1.0000.5503) foram iniciadas as atividades clínicas no Centro de Práticas Supervisionadas da Faculdade de Ciências da Saúde da Universidade do Vale do Paraíba. Os pesquisadores responsáveis comprometeram-se a seguir as normas preconizadas pela Resolução do CNS 466/12 e suas complementares, que tratam dos aspectos éticos e legais da pesquisa envolvendo seres humanos.

Trata-se de caso clínico de paciente com acometimento neurálgico pós-herpes zoster em diversas regiões do corpo, que apresentava condições de cooperar com o protocolo estabelecido pelo estudo. Antes do início do tratamento, o paciente fez a leitura e assinatura do Termo de Consentimento Livre e Esclarecido - TCLE, tomando ciência dos passos do protocolo clínico a ser aplicado e da eventual publicação dos resultados obtidos, resguardando sua identidade.

\section{Caracterização da paciente}

Paciente do gênero feminino, 41 anos, residente na cidade de São José dos Campos - São Paulo, Brasil, diagnosticada com Herpes zoster em abril de 2019, apresentando lesões em região torácica anterior (inframamária). Foi submetida ao tratamento padrão com aciclovir (via oral) e analgésicos, para diminuição da carga viral e melhora da dor, respectivamente. Negou doenças prévias e quaisquer tipos de tratamento médico no momento do início do atendimento. Relatou cicatrização das lesões em aproximadamente 3 semanas após o início das lesões cutâneas. Posteriormente à cicatrização das lesões causadas pelo $\mathrm{HZ}$, informou que sentia frequentes dores na região afetada e relatou que houve retardo no diagnóstico de $\mathrm{NPH}$ (Neuralgia pós-herpética), tanto pelo tempo decorrido, que o dissociava da $\mathrm{HZ}$, quanto pelos sintomas nem sempre específicos. Quando procurou nosso serviço, em setembro de 2019 , relatava dor intensa em região acometida pelo $\mathrm{HZ}$ principalmente quando se movimentava. Relatou, ainda, que no momento do atendimento utilizava 
dipirona, pois fármacos mais potentes prejudicavam suas atividades diárias e interferiam diretamente em sua qualidade de vida.

\section{Protocolo da Fotobiomodulação}

O protocolo de PBM constou de irradiações pontuais ao longo do trajeto do nervo acometido pelo Herpesvírus, com distância de $2 \mathrm{~cm}$ entre cada ponto, medida com o auxílio de uma régua. Para demarcação dos pontos na pele, foi utilizada uma caneta hipoalergênica resistente à umidade. Foi utilizado um aparelho laser, denominado cluster, com três lasers de GaAlAs (DMC, Brasil) com comprimento de onda de 660 $\mathrm{nm}$ (vermelho) e potência de $100 \mathrm{~mW}$. O tratamento contou com um total de 10 sessões, intervalo mínimo de 36 horas entre cada uma, durante 4 semanas. Os parâmetros de irradiação utilizados estão apresentados na Tabela 1.

Tabela 1 - Parâmetros de irradiação da PBM utilizados no presente estudo.

Parâmetros de aplicação da PBM

\begin{tabular}{lc}
\hline Comprimento de onda $(\mathrm{nm})$ & 660 (vermelho) \\
Potência do equipamento $(\mathrm{mW})$ & 100 \\
Tempo de irradiação por ponto (segundo) & 40 \\
Energia por ponto $(\mathrm{J})$ & 4 \\
Diâmetro do feixe $\left(\mathrm{cm}^{2}\right)$ & 0,1 \\
Número de pontos & 6 \\
Total de sessões & 10 sessões \\
\hline
\end{tabular}

Fonte: Elaborado pelos autores.

\section{Protocolo da Fotobiomodulação}

Para avaliar a qualidade de vida foi aplicado o questionário de Avaliação de Qualidade de Vida SF-36 (Short-Form Health Survey), instrumento que permite avaliar a Qualidade de Vida (QV) de forma multidimensional, desenvolvido em 1992 por Ware e Sherbourne em 1992 e validado no Brasil por Ciconelli et al., 1999.

O questionário SF-36 é amplamente utilizado em pesquisas na área da saúde e foi traduzido e adaptado para a população brasileira segundo a metodologia internacionalmente aceita. Esse instrumento é composto por 36 itens englobados em oito escalas ou componentes: capacidade funcional, aspectos físicos, dor, estado geral de saúde, vitalidade, aspectos sociais, aspectos emocionais, saúde mental e mais uma questão de avaliação comparativa entre as condições de saúde atual e a do período anterior. Avalia tanto aspectos negativos de saúde (doença ou enfermidade) quanto os aspectos positivos (bem-estar).

A avaliação dos resultados obtidos com a aplicação do instrumento SF-36 é realizada em duas fases. A primeira fase é de ponderação dos dados. As respostas de cada item são ponderadas com um valor pré-determinado e recebem uma pontuação de acordo com os autores do questionário. A segunda fase é o cálculo de Raw Scale. Nesta fase os valores são transformados em notas dos 8 domínios, que 
variam de 0 a 100, cujo valor obtido não apresenta nenhuma unidade. Quanto mais próximo ao valor 0 , pior é a qualidade de vida, e quanto mais próximo a 100 , melhor é a qualidade de vida.

Seguindo essas premissas, o SF-36 foi aplicado em dois momentos distintos, antes da aplicação do protocolo ( $1^{\mathrm{a}}$ sessão) e após o término do tratamento (10 sessão). Os resultados obtidos foram avaliados pela atribuição de escores para cada questão, os quais foram transformados em escala de zero (0) a cem (100).

\section{Avaliação da dor referida pelo instrumento EVA}

Para avaliação do nível de dor, foi utilizada a Escala Visual Analógica da Dor (EVA), instrumento unidimensional empregado para avaliação da intensidade da dor, largamente utilizada em pesquisas e atendimentos a pacientes. Trata-se de uma régua com as extremidades numeradas de $0-10$. Em uma extremidade da linha é marcada "nenhuma dor" e na outra "pior dor imaginável". Foi solicitado à paciente para avaliar e marcar na régua a dor percebida naquele momento. A EVA foi apresentada à paciente antes do início de cada uma das sessões de PBM, conforme previsto no protocolo clínico.

\section{Avaliação da algometria pelo instrumento Analgesímetro Digital}

Para avaliar quantitativamente a dor (algometria), foi utilizado um instrumento denominado analgesímetro digital (EFF 301, INSIGHT). Esse aparelho avalia a sensibilidade à dor a partir da pressão positiva exercida pela ponteira do sensor no local de dor referido pelo paciente, resultando em um valor quantificado em gramas (g). Ressalta-se que quanto maior for o limiar de dor, menor é a tolerância do ponto de pressão, e quanto menor for o limiar de dor, maior será a tolerância do ponto de pressão.

A paciente foi instruída a sinalizar ao pesquisador quando começasse a sentir a dor no local em que era exercida a pressão e, também, quando a dor se tornava intolerável. Ao sinalizar a dor, a ponteira era imediatamente retirada da referida região, e o valor em gramas apresentado no visor do equipamento era registrado para posterior análise. Essa avaliação foi realizada em cada uma das 10 sessões estabelecidas no protocolo clínico, sendo que a primeira avaliação foi realizada antes de seu início e a última após o término do protocolo com PBM.

\section{Análise estatística}

Os dados obtidos foram digitados, tabulados e analisados com o auxílio do programa Microsoft Excel $\circledast$, utilizando-se o teste $T$ pareado para avaliação de possíveis efeitos da PBM, adotando nível de significância de $5 \%(p<0,05)$, visando comprovar existência de diferença estatística significativa, antes e após a aplicação da PBM. Para melhor análise dos dados obtidos pelo SF-36, antes ( $1^{a}$ sessão) e após o tratamento $\left(10^{\mathrm{a}}\right.$ sessão) foi realizada análise descritiva visando comparar, de forma isolada, cada domínio do instrumento.

\section{RESULTADOS}

Para análise do limiar de dor pela escala visual analógica (EVA), o período do tratamento com PBM foi dividido em dois momentos, favorecendo a análise detalhada 
dos dados aferidos. Estabeleceu-se que o primeiro momento (início da PBM) correspondia aos valores obtidos entre a $1^{\text {a }}$ e $5^{a}$ sessões, enquanto o segundo momento (final da PBM), correspondia aos valores detectados entre a $6^{\mathrm{a}}$ e $10^{\mathrm{a}}$ sessões. A Tabela 2 e o Gráfico 1 apresentam os resultados da análise estatística dos dados, permitindo observar diferença significativa $(p<0,05)$ quando comparadas as médias dos valores do primeiro momento $(5 \pm 1,2)$ com as do segundo momento $(2,4$ $\pm 0,5)$.

Tabela 2- Resultados da dor referida obtidos com a Escala Visual Analógica (EVA) no primeiro momento do protocolo (entre a $1^{\text {a }}$ e $5^{\mathrm{a}}$ sessão) e no segundo momento do protocolo com PBM (entre

$6^{\mathrm{a}}$ e $10^{\mathrm{a}}$ sessão). Valor de $\boldsymbol{p}$ obtido a partir do teste T pareado, analisando os valores entre as sessões $(p<0,05)$.

\begin{tabular}{|c|c|c|c|c|}
\hline \multicolumn{5}{|c|}{ EVA - Escala Visual Analógica } \\
\hline \multicolumn{2}{|c|}{ Início da PBM } & \multicolumn{2}{|c|}{ Final da PBM } & \multirow{2}{*}{${ }^{*} p$} \\
\hline $\begin{array}{l}\text { № da } \\
\text { sessão }\end{array}$ & EVA & $\begin{array}{c}\text { № da } \\
\text { sessão }\end{array}$ & EVA & \\
\hline $1^{a}$ & 7 & $6^{\mathrm{a}}$ & 3 & \\
\hline $2^{\underline{a}}$ & 4 & $7^{\mathrm{a}}$ & 3 & \\
\hline $3^{\mathrm{a}}$ & 5 & $8^{\mathrm{a}}$ & 2 & \\
\hline $4^{\mathrm{a}}$ & 5 & $9 \underline{a}$ & 2 & \\
\hline $5^{\mathrm{a}}$ & 4 & $10^{\mathrm{a}}$ & 2 & \\
\hline Média $\pm \mathrm{D}$ & $5 \pm 1,2$ & & $2,4 \pm 0,5$ & 0,003 \\
\hline
\end{tabular}

Legenda: * ${ }^{*}=$ Valor de significância $<0,05 / \pm$ DP = Desvio padrão em relação à média. Fonte: Elaborado pelos autores.

Gráfico 1 - Gráfico representativo da média \pm DP (Desvio Padrão) da dor referida pela EVA quando comparados o primeiro momento do protocolo (entre a $1^{\mathrm{a}}$ e $5^{\mathrm{a}}$ sessão) ao segundo momento do protocolo com PBM (entre $6^{\mathrm{a}}$ e $10^{\mathrm{a}}$ sessão).

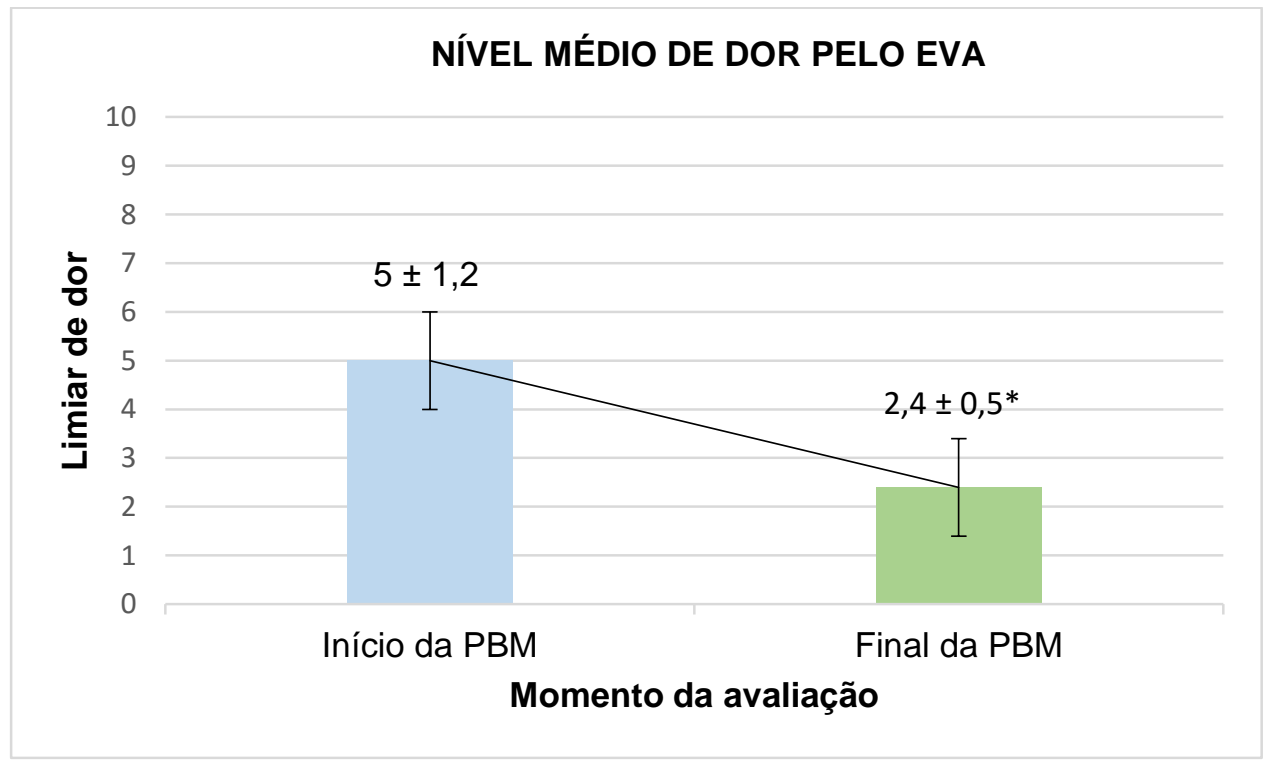

Legenda: \pm DP: Desvio padrão em relação a média / * $p=$ Valor de significância $<0,05$. Fonte: Elaborado pelos autores.

O questionário SF-36 foi aplicado antes do início da $1^{a}$ sessão e da $10^{a}$ sessão 
de tratamento com PBM, possibilitando avaliar a qualidade de vida do indivíduo nesses dois momentos distintos. Observou-se que todos os domínios apresentaram resultados positivos e podem ser apresentados na seguinte ordem, do mais expressivo para o menos expressivo: limitação por aspectos físicos, vitalidade, capacidade funcional, dor, aspectos emocionais, aspectos sociais, saúde mental e estado geral de saúde. Por ser relato de caso clínico de um único indivíduo, foi utilizada a análise descritiva visando evidenciar aspectos importantes em momentos distintos do protocolo de tratamento proposto (Tabela 3 e Gráfico 2).

Tabela 3 - Resultado da análise descritiva de Qualidade de vida (SF-36), classificada por domínios, no momento inicial (pré-terapia) e no momento final (pós-terapia). Resultados obtidos a partir da pontuação do domínio correspondente na $1^{\text {a }}$ sessão, extraindo a diferença em porcentagem (\%) comparados com a $10^{a}$ sessão. $O$ índice de significância de $p<0,001$ foi obtido comparando-se as médias $( \pm \mathrm{DP})$ da $1^{\mathrm{a}}$ sessão com as da $10^{\mathrm{a}}$ sessão, considerando todos os domínios avaliados.

\begin{tabular}{|c|c|c|c|c|}
\hline \multicolumn{5}{|c|}{ SF- 36} \\
\hline Domínios & 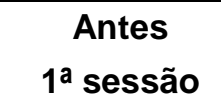 & $\begin{array}{c}\text { Depois } \\
10^{\mathrm{a}} \text { sessão }\end{array}$ & $\begin{array}{c}\text { Diferença } \\
\%\end{array}$ & ${ }^{*} p$ \\
\hline Capacidade funcional & 30 & 70 & 40 & \\
\hline Limitação por Aspectos Físicos & 0 & 55 & 55 & \\
\hline Dor & 10 & 50 & 40 & \\
\hline Estado Geral de Saúde & 55 & 70 & 15 & \\
\hline Vitalidade & 20 & 65 & 45 & \\
\hline Aspectos Sociais & 25 & 50 & 25 & \\
\hline Aspectos Emocionais & 32 & 70 & 38 & \\
\hline Saúde Mental & 50 & 68 & 18 & \\
\hline
\end{tabular}

Legenda: ${ }^{*} \mathbf{p}=$ Valor de significância $<0,05 / \pm \mathrm{DP}=$ Desvio padrão em relação à média. Fonte: Elaborado pelos autores (2021).

Gráfico 2 - Figura representativa dos dados obtidos em cada domínio pela aplicação do instrumento SF-36, de qualidade de vida, antes ( $1^{\mathrm{a}}$ sessão) e após o término do tratamento com PBM (10 ${ }^{\text {a }}$ sessão).

\section{Qualidade de vida antes e após as sessões pela PBM}

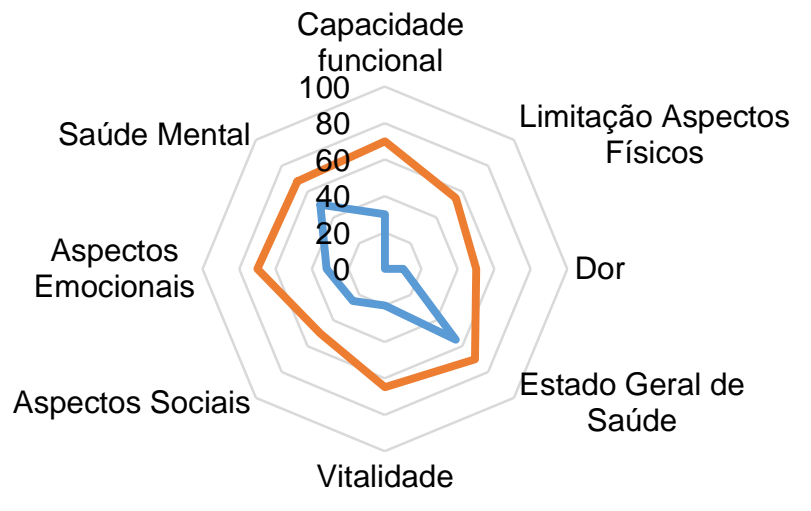

Antes Depois

Fonte: Elaborado pelos autores. 
$\mathrm{Na}$ Tabela 4 são apresentados os valores do limiar de dor da paciente, obtidos com o auxílio do analgesímetro digital. A comparação das médias $( \pm \mathrm{DP})$ dos dados obtidos na $1^{\text {a }}$ sessão (antes PBM) com os da $10^{a}$ sessão (após PBM) apresentou diferença significativa $(p<0,039)$, evidenciando aumento na tolerância a dor nos pontos de pressão após a terapia.

Tabela 4 - Dados obtidos pelo analgesímetro digital relacionados ao limiar de dor. Resultados obtidos a partir do teste de t pareado, comparação do limiar de dor referida na $1^{\text {a }}$ sessão e na $10^{a}$ sessão.

Algometria aferida pelo Analgesímetro Digital

\begin{tabular}{|c|c|c|c|}
\hline \multirow{2}{*}{$\begin{array}{c}\text { Pontos de Pressão } \\
\text { no do ponto } \\
\end{array}$} & \multicolumn{3}{|c|}{ Valor em gramas $(\mathrm{g})$} \\
\hline & 1 a Sessão & 10a sessão & ${ }^{*} p$ \\
\hline 1 & 213,4 & 350,3 & \\
\hline 2 & 173,4 & 254,6 & \\
\hline 3 & 226,8 & 246,3 & \\
\hline 4 & 248,0 & 289,4 & \\
\hline 5 & 107,6 & 213,8 & \\
\hline 6 & 217,1 & 177,2 & \\
\hline Média \pm DP & $197,7 \pm 50,42$ & $255,2 \pm 60,15$ & ${ }^{*} 0,039$ \\
\hline
\end{tabular}

\section{DISCUSSÃO}

A anamnese inicial da paciente do presente caso clínico permitiu verificar que a $\mathrm{NPH}$ acarretou prejuízos intensos em sua qualidade de vida. Estudos relacionados às pesquisas sobre NPH referem essa dor como causa incapacitante aos seus portadores, por interferir diretamente no desenvolvimento de atividades do cotidiano e, consequentemente, na qualidade de vida. Estão afetados tanto aspectos físicos quanto emocionais, tais como os relacionados a distúrbios do sono, dor com ou sem contato físico, frustração e, em alguns casos, até depressão (GOMES et al., 2018; MIZUKAMI et al., 2018).

Em estudo recente, desenvolvido por Mukhtar et al. (2020), foi realizado tratamento com PBM em 15 participantes diagnosticados com NPH (16 sessões, 8 semanas, entre início e fim do tratamento). Os pesquisadores apontaram redução da dor ao final do protocolo de tratamento, resultados semelhantes aos observados neste caso clínico, que também verificou redução na dor avaliada pela EVA.

Neste estudo foram empregados três instrumentos de coleta de dados, dois relacionados à avaliação da intensidade da dor e o terceiro aplicado para avaliar quantos e quais domínios da qualidade de vida estavam afetados pela NPH, permitindo que essas análises fornecessem maior confiabilidade aos resultados aqui relatados decorrentes a PBM para tratamento da NPH.

Como já relatado em estudos que utilizaram PBM em pacientes portadores de $\mathrm{NPH}$, essa é uma terapia segura, tanto para os profissionais quanto para os pacientes, com riscos e efeitos colaterais mínimos, desde que utilizados os parâmetros adequados (GOMES et al., 2018). 
A PBM é apresentada como uma modalidade terapêutica válida quando aplicada em paciente portador de NPH, tendo em vista seus efeitos analgésico, antiinflamatório e antiedematoso, que por sua vez pode reduzir a sintomatologia da dor, além da reparação tecidual e melhora da perfusão tecidual pela vasodilatação (DE CARVALHO et al., 2009).

Observou-se, no presente caso clínico, que a paciente passou por dificuldades para o diagnóstico inicial da NPH, visto que as dores, que surgiram dois meses após a cura das erupções, foram associadas à outras causas. Estudos anteriores relataram que, em virtude do início da NPH apresentar grande variação temporal, que varia de mês até anos após a ocorrência das erupções cutâneas, o diagnóstico correto pode dispender tempo prolongado, acarretando piora no quadro álgico (COELHO et al., 2014; OLIVEIRA, et al., 2016).

Vasconcelos Filho et al. (2012), em estudo de caso relatando os resultados obtidos com o uso da PBM em paciente com NPH, pós transplante pulmonar, atestaram a eficácia dessa ferramenta terapêutica pela melhora significativa da resposta álgica, trazendo benefícios e redução do uso de fármacos, sendo essa uma importante vantagem da PBM.

O tratamento convencional da neuralgia pós-herpética contempla o uso de diversos fármacos tais como antidepressivos, anticonvulsivantes, opiáceos e antiinflamatórios. Estudos demonstraram que o uso desses fármacos apresentam efeitos colaterais que afetam a qualidade de vida do paciente (DUBINSKY et al., 2004; DWIGHT et al., 2019).

A NPH é um acometimento, na maioria das vezes, incapacitante devido a dor, afetando de alguma forma a qualidade de vida de seus portadores, interferindo em suas atividades diárias e desencadeando problemas em diversos aspectos (MIZUKAM et al., 2018; HWANG et al., 2018). No presente caso clínico, foi possível observar os efeitos da NPH e os prejuízos diretos à qualidade de vida da paciente, que relatou a falta de energia para realização das atividades diárias, tanto físicas quanto laborais e as relativa ao convívio social. Ressalta-se que após o tratamento com PBM a paciente não fez mais uso de analgésicos em virtude da redução significativa da dor.

O emprego da EVA, antes da aplicação do protocolo clínico em todas as sessões, permitiu quantificar a dor referida pela paciente, ao longo do período analisado. Isso permitiu o acompanhamento contínuo de seu limiar de dor e demonstrar a redução significativa da dor na última sessão.

O questionário de qualidade de vida SF-36 permitiu obter visão holística individualizada do impacto da NPH na paciente atendida, fornecendo dados referentes aos oito domínios que refletem a qualidade de vida: capacidade funcional, limitação por aspectos físicos, dor, estado geral de saúde, vitalidade, aspectos sociais, aspectos emocionais e saúde mental. A partir da análise descritiva observou-se melhora em todos os domínios, sendo que o quesito limitação por aspectos físicos apresentou resultado expressivo (55\%), bem como vitalidade, capacidade funcional, dor e aspectos emocionais, quando comparados os dados da primeira à última sessão.

Estatisticamente, foi observada significante melhora $(p=0,001)$ na qualidade de vida da paciente, quando comparados os domínios avaliados antes e após a PBM. A paciente do presente caso clínico relatou sensação de bem-estar, que permitiu que ela retomasse suas atividades diárias com mais disposição, demonstrando a ação positiva do tratamento com PBM na NPH.

A algometria tem sido usualmente utilizada quanto à quantificação da dor, permitindo identificar e acompanhar a evolução do limiar de dor de um paciente com 
maior precisão ao longo de um protocolo de tratamento (PIOVESAN et al., 2001). Sua aplicação no presente estudo possibilitou evidenciar diferença significativa $(p=0,039)$ quando comparada a $1^{\text {a }}$ sessão (início da $\mathrm{PBM}$ ) à $10^{\mathrm{a}}$ sessão (término da $\mathrm{PBM}$ ). Observou-se melhora expressiva do início da PBM $(197,7 \pm 50,42)$ e término da PBM $(255,2 \pm 60,15)$, demonstrando a eficácia do protocolo. Ressalta-se que o valor obtido era referente à sensação de dor em cada ponto de pressão exercida pela ponteira do equipamento sobre a região acometida pela NPH.

A NPH merece maior visibilidade nas ciências médicas, visto que o problema é real e afeta, principalmente, a população idosa e os pacientes imunodeprimidos, mais sensíveis aos prejuízos em sua qualidade de vida. Esses fatos incentivam a realização de novos estudos, com número ampliado de pacientes, buscando validar a eficácia do emprego da PBM no tratamento da NHP.

\section{CONCLUSÃO}

Os resultados do presente caso clínico sugerem que o protocolo clínico da PBM foi eficaz no tratamento de NPH, com evidente redução da dor e melhora da qualidade de vida. A utilização dos instrumentos EVA, Analgesímetro Digital e do Questionário SF-36 permitiram a aquisição de dados quantitativos fundamentais para análise da evolução dessa paciente, antes e após o tratamento.

\section{AGRADECIMENTO}

Agradecimento à Coordenação de Aperfeiçoamento de Pessoal de Nível Superior - CAPES -Código de financiamento 001, pelo apoio financeiro.

\section{REFERÊNCIAS}

BRASIL Ministério da Saúde. Secretaria de Vigilância em Saúde. CoordenaçãoGeral de Desenvolvimento da Epidemiologia em Serviços. Guia de Vigilância em Saúde: volume único. 3a․ ed. Brasília, DF.: Ministério da Saúde, 2019.

CAVALCANTI, M.F. et al. Comparative study of the physiotherapeutic and drug protocol and low-level laser irradiation in the treatment of pain associated with temporomandibular dysfunction. Photomed. laser surg., v. 34, n. 12, p. 652-656, 2016.

CHEN, N. et al. Antiviral treatment for preventing nerve pain after shingles (postherpetic neuralgia). Cochrane Database Syst Rev., v. 2, n. 1, p. 1-10, 2014.

CICONELLI, R. M. et al. Tradução para a língua portuguesa e validação do questionário genérico de avaliação de qualidade de vida SF-36 (Brasil SF-36). Rev. bras. reumatol., v. 39, n. 3, p. 143-50, 1999.

COELHO, P. A. B. et al. Diagnosis and management of herpes zoster by the family and community physician. Rev. Bras. Med. Fam. Comunidade, v. 9, n. 32, p. 279285, 2014.

DE CARVALHO, F. et al. Impact of low-intensity laser on the suppression of infections caused by Herpes simplex viruses 1 and 2: in vitro study. Rev. Soc. Bras. 
Med. Trop., v. 42, n. 1, 2009.

DING, X.D. et al. Botulinum as a toxin for treating post-herpetic neuralgia. Iran. j. public health, v. 46, n. 5, p. 608, 2017.

DUBINSKY, R. M. et al. Practice Parameter: Treatment of postherpetic neuralgia:[RETIRED]: An evidence-based report of the Quality Standards Subcommittee of the American Academy of Neurology. Neurology, v. 63, n. 6, p. 959-965, 2004.

DWIGHT, E. et al. Intravenous lidocaine in the management of chronic peripheral neuropathic pain: a randomized-controlled trial. Can. J. Anaesth., v. 66, n. 7, p. 820827, 2019.

GOMES, R.N.S. et al. Efeitos da fotobioestimulação no tratamento da neuralgia pósherpética: relato de caso. Rev. Bras. Geriatr. Gerontol., Rio de Janeiro, v. 21, n. 1, 105-110. 2018.

HWANG, S. et al. Neuropathic pain: A patient-centred approach to measuring outcomes. Health expect., v. 21, n. 4, p. 774-786, 2018.

MIZUKAMI, A. et al. Impact of herpes zoster and post-herpetic neuralgia on healthrelated quality of life in Japanese adults aged 60 years or older: results from a prospective, observational cohort study. Clin. drug investg., v. 38, n. 1, p. 29-37, 2018.

MUKHTAR, R. et al. Role of low-level laser therapy in post-herpetic neuralgia: a pilot study. Lasers med. sci., p. 1-6, 2020.

NEVES, M.F. et al. Long-term analyses of spastic muscle behavior in chronic poststroke patients after near-infrared low-level laser therapy $(808 \mathrm{~nm})$ : a doubleblinded placebo-controlled clinical trial. Lasers med. Sci., v. 35, p. 1459-1467, 2020. https://doi.org/10.1007/s10103-019-02920-3.

OLIVEIRA, C. A.; CASTRO, A. P. C. R.; MIYAHIRA, S. A. Post-herpetic neuralgia. Rev. Dor, v. 17 , p. 52-55, 2016.

PIOVESAN, E. J. et al. Utilização da algometria de pressão na determinação dos limiares de percepção dolorosa trigeminal em voluntários sadios: um novo protocolo de estudos. Arq. neuropsiquiatr., v. 59, n. 1, p. 92-96, 2001.

PORTELLA, A. V. T.; SOUZA, L. C. B.; GOMES, J. M. A. Herpes-zoster and postherpetic neuralgia. Rev Dor, v. 14, n. 3, p. 210-5, 2013.

LE, P.; ROTHBERG, M. Herpes zoster infection. Bmj, v. 364, 2019. doi: 10.1136/bmj.k5095.

SANTOS, J.A.F. et al. Effects of Low-Power Light Therapy on the Tissue Repair Process of Chronic Wounds in Diabetic Feet. Photomed. laser surg., v. 36, n. 6, p. 
298-304, 2018.

VASCONCELOS FILHO, P.O.V. et al. Tratamento alternativo para neuralgia pósherpética após transplante pulmonar. Relato de caso. Rev. Dor, v. 13, n. 1, p. 85-88, 2012.

WARE, J. E.; SHERBOURNE, C. D. The MOS 36-item short-form health survey (SF36): I. Conceptual framework and item selection. Med. Care, v. 30, n. 6, p. 473-483, 1992.

YAWN, B. P.; GILDEN, D. The global epidemiology of herpes zoster. Neurology, v. 81, n. 10, p. 928-930, 2013.

YOUNGER, J.; MCCUE, R.; MACKEY, S. Pain outcomes: a brief review of instruments and techniques. Curr. pain headache rep., v. 13, n. 1, p. 39-43, 2009. 